\title{
SPATIALLY RESOLVED ULTRAVIOLET, H $\alpha$, INFRARED, AND RADIO STAR FORMATION IN M81
}

\author{
K. D. Gordon, ${ }^{1}$ P. G. Pérez-González, ${ }^{1}$ K. A. Misselt, ${ }^{1}$ E. J. Murphy, ${ }^{2}$ G. J. Bendo, ${ }^{1}$ F. Walter, ${ }^{3}$ M. D. Thornley ${ }^{4}, 5$ \\ R. C. Kennicutt, JR., ${ }^{1}$ G. H. Rieke, ${ }^{1}$ C. W. Engelbracht, ${ }^{1}$ J.-D. T. Smith, ${ }^{1}$ A. Alonso-Herrero, ${ }_{1}^{1}$ P. N. Appleton,${ }^{6}$ \\ D. Calzetti, ${ }^{5}$ D. A. Dale, ${ }^{7}$ B. T. Draine, ${ }^{8}$ D. T. Frayer, ${ }^{6}$ G. Helou, ${ }^{6}$ J. L. Hinz, ${ }^{1}$ D. C. Hines, ${ }^{1,9}$ D. M. Kelly, ${ }^{1}$ \\ J. E. Morrison, ${ }^{1}$ J. Muzerolle, ${ }^{1}$ M. W. Regan, ${ }^{5}$ J. A. Stansberry, ${ }^{1}$ S. R. Stolovy, ${ }^{6}$ \\ L. J. Storrie-Lombardi, ${ }^{6}$ K. Y. L. Su, ${ }^{1}$ and E. T. Young ${ }^{1}$ \\ Received 2004 March 26; accepted 2004 May 20
}

\begin{abstract}
We present Multiband Imaging Photometer for Spitzer (MIPS) observations of M81 at 24, 70, and $160 \mu \mathrm{m}$. The grand design nature of M81 is clearly seen, showing two well-resolved spiral arms containing numerous bright star-forming regions. The MIPS images reveal a significant amount of cold dust associated with the spiral arms. We investigate the variation of the ultraviolet (UV), $\mathrm{H} \alpha$, and infrared (IR) luminosities and star formation rate (SFR) indicators across the face of M81 using the MIPS images and archival UV and $\mathrm{H} \alpha$ images. For regions in M81, we find that UV and $\mathrm{H} \alpha$ SFRs (uncorrected for dust attenuation) are always lower than the IR SFR. The cause of this behavior is dust attenuation and/or using SFR calibrations appropriate for entire galaxies, not regions in galaxies. The characteristics of the dust attenuation for the regions indicate the dust grains and/or geometry are different from those in starburst galaxies. The behavior of the infrared-radio correlation in M81 is seen to vary from the global average, with variations correlated with the morphology of M81.
\end{abstract}

Subject headings: dust, extinction — galaxies: individual (M81) — galaxies: ISM — galaxies: spiral infrared: galaxies

\section{INTRODUCTION}

The galaxy M81 (NGC 3031) is one of the largest grand design spiral galaxies in the sky $\left(14^{\prime} \times 27^{\prime}\right)$. It is a member of the M81 galaxy group, whose principle members are M81, M82, NGC 3077, and NGC 2976 and which includes a number of dwarf galaxies (Appleton et al. 1981; Boyce et al. 2001). Prominent tidal $\mathrm{H}$ I tails around M81 show that it is interacting with M82 and NGC 3077 (Yun et al. 1994). The Cepheid distance to M81 is $3.6 \mathrm{Mpc}$ (Freedman et al. 2001). The proximity of M81 has made it a favorite target for many investigations of galaxy properties from the X-ray to radio (e.g., Kaufman et al. 1987; Devereux et al. 1995; Hill et al. 1995; Kong et al. 2000; Swartz et al. 2003).

Many well-resolved images of M81 from ultraviolet (UV) to radio wavelengths have been taken, with the notable exception of the infrared (IR). Previously, well-resolved far-IR images of galaxies have only been possible for Local Group galaxies such as the Magellanic Clouds (Braun et al. 1998; Wilke et al. 2003), M31 (Haas et al. 1998), and M33 (Hippelein et al. 2003). With the successful launch of the Spitzer Space Telescope (Werner et al. 2004), it is now possible to map many large galaxies in the far-IR with good spatial resolution, good sensitivity, and in a reasonable amount of time. Infrared Array Camera (IRAC; Fazio et al. 2004) and the Multiband Imaging

\footnotetext{
1 Steward Observatory, University of Arizona, Tucson, AZ 85721.

2 Department of Astronomy, Yale University, New Haven, CT 06520.

3 NRAO, P.O. Box O, Socorro, NM 87801.

4 Department of Physics, Bucknell University, Lewisburg, PA 17837.

5 Space Telescope Science Institute, Baltimore, MD 21218.

6 Spitzer Science Center, California Institute of Technology, Pasadena, CA 91125.

7 Department of Physics and Astronomy, University of Wyoming, Laramie, WY 82071.

${ }^{8}$ Princeton University Observatory, Princeton, NJ 08544.

9 Space Science Institute, Boulder, CO 80301.
}

Photometer for Spitzer (MIPS; Rieke et al. 2004) images of M81 were taken as part of the commissioning of Spitzer and showcased in the first Spitzer press release. The MIPS images are presented in this paper, and Willner et al. (2004) presents the IRAC images. M81 is one of the key galaxies in the Spitzer Infrared Nearby Galaxies Survey (SINGS; Kennicutt et al. 2003).

We have chosen to investigate the variation of star formation rate (SFR) indicators and the infrared-radio correlation across the disk of M81 to highlight two of the questions that can be probed with these new observations. We compare the MIPS observations with existing UV and $\mathrm{H} \alpha$ images to probe the behaviors of the IR, $\mathrm{H} \alpha$, and UV SFR indicators across M81. Such comparisons of SFR indicators have been made for global galaxy fluxes (e.g., Bell \& Kennicutt 2001; Kewley et al. 2002; Rosa-González et al. 2002), but rarely has it been possible to resolve all three SFR indicators in a single galaxy. By studying the resolved behavior of these SFR indicators, a better understanding of their sensitivities to dust and stellar age can be determined. This will improve the accuracy of these SFR indicators for both resolved and global galaxy measurements. Radio emission is another possible SFR indicator, but it is not as mature as the others (Haarsma et al. 2000; Bell 2003). As such, we have chosen to explore the more established infrared-radio correlation (Helou et al. 1985; Condon 1992; Yun et al. 2001; Pierini et al. 2003).

\section{DATA \\ 2.1. MIPS Observations}

Images of M81 at 24, 70, and $160 \mu \mathrm{m}$ (Spitzer AOR key 0007864576) were obtained in a manner similar to that described in Engelbracht et al. (2004) for NGC 55. The MIPS images were reduced using the MIPS Instrument Team Data Analysis Tool (Gordon et al. 2004) as described in Engelbracht et al. (2004). The uncertainties on the final absolute calibrations 
are estimated at $10 \%, 20 \%$, and $20 \%$ for the 24,70 , and $160 \mu \mathrm{m}$ data, respectively. The final MIPS mosaics are displayed in Figure 1 (Plate 1). The 70 and $160 \mu \mathrm{m}$ images exhibit linear streaks along the scan direction (roughly north-south), which are residual instrumental artifacts caused by the time-dependent responsivity of the Ge detectors.

\subsection{Ancillary Data}

Ultraviolet images of M81 were taken with the Ultraviolet Imaging Telescope in far-UV and near-UV bands (Stecher et al. 1997; Marcum et al. 2001). The deepest, geometrically corrected, flux-calibrated images for each band were retrieved from the Multimission Archive at Space Telescope (MAST). The $\mathrm{H} \alpha$ image of M81 was taken from Greenawalt et al. (1998). The radio image at $20 \mathrm{~cm}$ was created from archival Very Large Array (VLA) C-array data, using standard AIPS reduction techniques.

\subsection{Resolution Matching}

Each image of M81 was cropped to a common field of view $\left(20^{\prime} \times 30^{\prime}\right)$ and convolved to match the point-spread function (PSF) of the MIPS $160 \mu \mathrm{m}$ image to allow for a consistent comparison of the images. We used a Fourier transform technique for the convolution as described in Engelbracht et al. (2004). All of the convolved images are shown in Figure 2 (Plate 2). The $160 \mu \mathrm{m}$ PSF clearly has an Airy ring resulting in a prominent ring surrounding point sources in some of the images.

\section{RESULTS}

\subsection{Morphology}

At $160 \mu \mathrm{m}$ resolution, the grand design nature of M81 is very prominent in all but the $R$-band image. The $R$-band image displays a morphology dominated by the bulge, with only faint spiral arms present. At the other wavelengths, the spiral arms show a continuum of morphologies from being a string of point sources $(\mathrm{H} \alpha$ and $24 \mu \mathrm{m})$ to exhibiting both point sources and an underlying smoother distribution (UV and $70 \mu \mathrm{m}$ ) to fairly smooth arms with faint point sources (160 $\mu \mathrm{m}$ and radio). This difference in the appearance is related to the age of stars probed and the importance of dust attenuation. For example, $\mathrm{H} \alpha$ probes younger star formation than the UV images and, as a result, the UV images are smoother than the $\mathrm{H} \alpha$ image. Evidence that dust attenuation is affecting the arm morphology is seen in the southeast of M81, where a prominent piece of the galaxy is missing from the UV and $\mathrm{H} \alpha$ images (see ellipse in Fig. 2), but not from the IR or radio images.

\subsection{Star Formation Rate Indicators}

We investigated the behavior of the popular $\mathrm{UV}, \mathrm{H} \alpha$, and IR SFRs in M81. H $\alpha$ and UV SFR indicators suffer from dust attenuation and probe stellar ages of 10 and $100 \mathrm{Myr}$, respectively. The IR SFR indicator does not have an age bias but does assume that all of the starlight from the star formation is absorbed by dust and reemitted in the IR. As we use resolved images of M81 for all three SFR indicators, we can probe the biases affecting each method in detail. We have extracted fluxes for specific regions in M81 from the $160 \mu \mathrm{m}$ resolution images, correcting the UV and $\mathrm{H} \alpha$ fluxes for a foreground Milky Way extinction with $E(B-V)=0.08$ (Schlegel et al. 1998). The regions are shown in Figure 3.

In order to convert measured $\mathrm{UV}, \mathrm{H} \alpha$, and IR luminosities to SFRs, calibrations are needed. One way to determine such

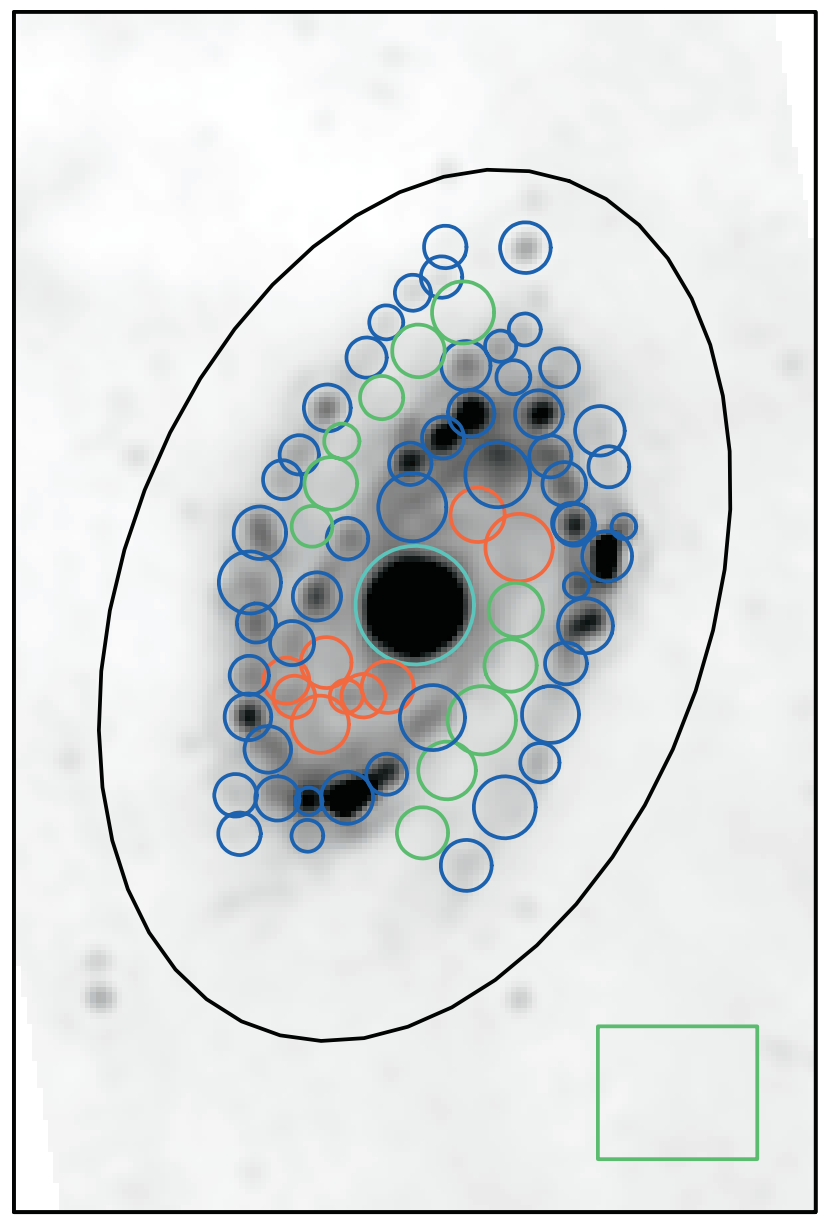

FIG. 3.-Regions for which fluxes were extracted shown superimposed on the M81 $24 \mu \mathrm{m}$ image. The size of the extraction aperture is reflected in the size of the circle for each region. The average diameter of the apertures is $1.2 \mathrm{kpc}$. The color coding of the regions is given in Fig. 4. The green box in the lower right corner gives the sky aperture. At $70 \mu \mathrm{m}$, the sky noise contribution to the uncertainty in the average aperture is $\sim 2 \mathrm{mJy}$. This shows that the linear streaks are not affecting the $70 \mu \mathrm{m}$ aperture measurements as all the fluxes are above $80 \mathrm{mJy}$. The field of view of the image is $20^{\prime} \times 30^{\prime}$.

calibrations is to use stellar evolutionary synthesis models (e.g., Fioc \& Rocca-Volmerange 1997; Leitherer et al. 1999). Kennicutt (1998) has provided such calibrations with stellar age assumptions appropriate for entire galaxies. While the Kennicutt (1998) calibrations will be invalid at some level for regions of galaxies, we have chosen to use them as they allow a physical basis for interpretation of luminosities. The total IR flux was calculated using the TIR equation of Dale \& Helou (2002) formulated specifically to determine the $3-1100 \mu \mathrm{m}$ luminosity given MIPS measurements. All of the SFRs in this paper are uncorrected for dust attenuation as proper treatment of dust attenuation is beyond the scope of this paper (e.g., Witt \& Gordon 2000).

The SFRs for the whole galaxy are estimated to be $0.31,0.38$, and $0.89 M_{\odot} \mathrm{yr}^{-1}$ from the integrated UV, $\mathrm{H} \alpha$, and IR luminosities, respectively. The luminosities and SFRs determined in the regions defined in Figure 3 are plotted in Figure 4. The arm regions display the largest area-normalized IR SFRs, which is evidence that they have the highest SFRs. The ratio of $\mathrm{H} \alpha / \mathrm{IR}$ luminosities is higher for the arm regions than any of the other regions. For all the regions, the UV and $\mathrm{H} \alpha$ SFRs are always smaller than the IR SFRs and there is a weak trend toward smaller UV/TIR and H $\alpha /$ TIR SFR ratios as the TIR SFR 

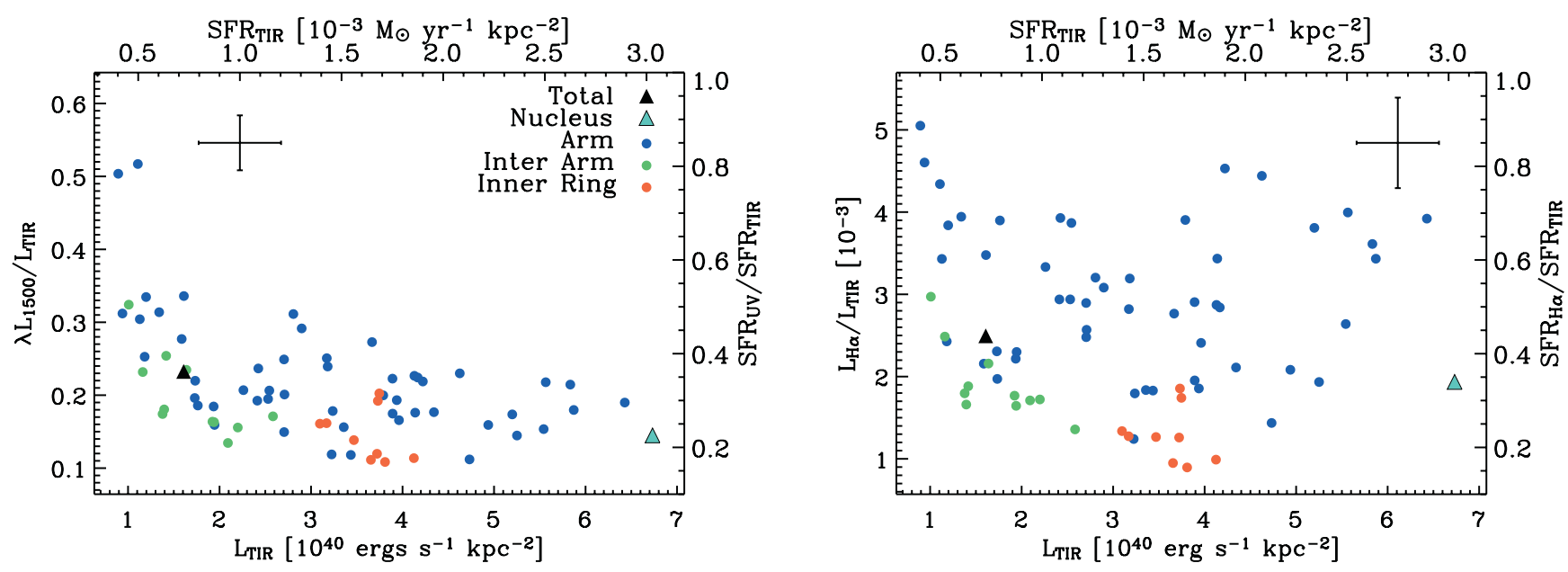

FIG. 4.-Luminosities for UV and $\mathrm{H} \alpha$, ratiod to the IR luminosity, plotted vs. the IR luminosity. The IR luminosity is normalized to the area in kpc ${ }^{2}$ of each extraction aperture. The corresponding SFRs and SFR ratios are indicated on the opposite axes. Representative uncertainties are shown.

increases. There are three possible causes of these effects: (1) dust is attenuating the $\mathrm{UV}$ and $\mathrm{H} \alpha$ measurements; (2) a fraction of the IR flux may not be associated with recent star formation (i.e., dust heated by old stars); (3) the luminosity-SFR calibrations of Kennicutt (1998) are not appropriate for individual regions in a galaxy, where there may be transfer of energy between different regions (radiative transfer effects) and/or stellar age differences from those assumed by Kennicutt (1998), which will affect SFR indicators at different levels. We will investigate the relative importance of these factors in future papers but briefly discuss the first two points below.

The association of significant IR emission with bright UV and $\mathrm{H} \alpha$ regions is evidence for significant dust attenuation. The dust attenuation is probed in Figure 5, where the IR/UV ratio and UV color attenuation measures are plotted. The IR/UV ratio has been shown to be a good dust attenuation measure for galaxies and the UV attenuations for the extracted regions vary between 0.5 and $3.0 \mathrm{mag}$ for $\log \left(L_{\mathrm{TIR}} / \lambda L_{1500}\right)$ between 0.5 and 1.5 (Gordon et al. 2000). The $L_{2500} / L_{1500}$ ratio is also a dust

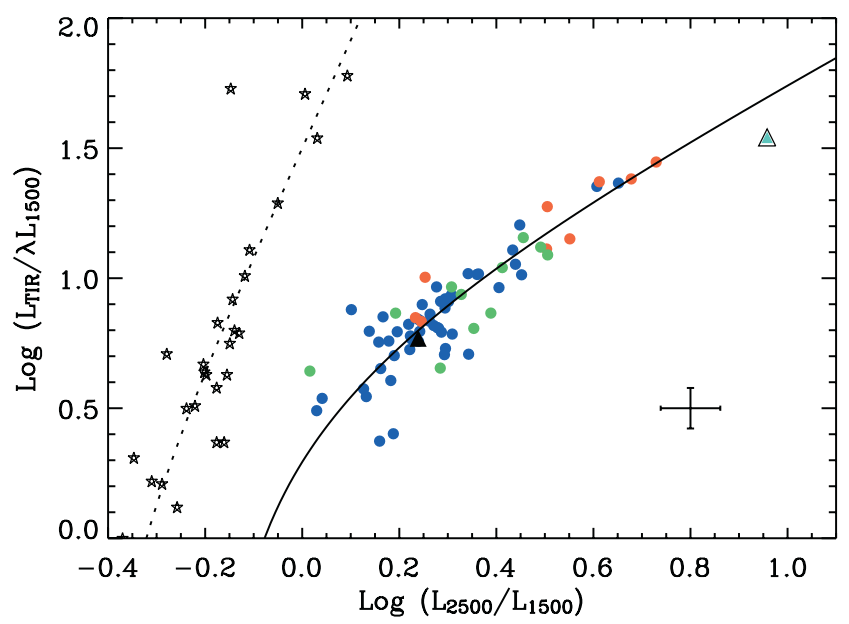

FIG. 5.-IR/1500 Å luminosity ratio plotted vs. the UV color $\left(L_{2500} / L_{1500}\right.$ ratio). The symbols are the same as in Fig. 4. The solid curve gives the linear fit $\left[L_{\mathrm{TIR}} / \lambda L_{1500}=-2.42+3.81\left(L_{2500} / L_{1500}\right)\right]$ to the M81 data. The star symbols give the observations for a sample of starburst galaxies, and the dotted line gives the Meurer et al. (1999) relationship determined for starburst galaxies. Representative uncertainties are shown. attenuation measure, but as it is a UV color, it is significantly more uncertain (Witt \& Gordon 2000). This flux ratio is very similar to the more familiar $\beta$ dust attenuation measure (Meurer et al. 1999). The starburst calibration of these measures is shown in Figure 5 and does not describe the measurements of M81. Deviations from the starburst calibration were also found by Bell et al. (2002) for Large Magellanic Cloud H II regions and by Kong et al. (2004) for global measurements of galaxies more quiescent than starbursts. This implies that the dust geometry and/or dust grain properties are different between starburst galaxies and star-forming regions in normal galaxies.

In studies of IR SFRs, it is common to assume that the cold dust is mainly heated by old stars, and thus the cold dust emission $(160 \mu \mathrm{m})$ should not be used in determining IR SFRs. The M81 images presented in this paper allow us to probe this assumption for the M81 disk but not the active galactic nucleus (AGN)-dominated nucleus. The morphology of the spiral arms progresses from pointlike to fairly smooth from 24 to $160 \mu \mathrm{m}$. The $\mathrm{H} \alpha$ and $\mathrm{UV}$ bright regions are seen at $160 \mu \mathrm{m}$ implying that young stars heat a fraction of the $160 \mu \mathrm{m}$ emission. The morphology of the IR images best matches the $\mathrm{H} \alpha$ and UV images as opposed to the $R$-band image (an old star tracer in M81), but this correlation is not enough to identify the dominant $160 \mu \mathrm{m}$ heating source, as the spiral density wave concentrates the dust in the arms. The smoother morphology of the UV images compared to the $\mathrm{H} \alpha$ image shows that the nonionizing UV bright stars have migrated out of their natal regions. Young, hot stars are more efficient than the old stars at heating dust since their emission peaks at $\mathrm{UV} /$ blue wavelengths where dust is most efficient at absorbing photons. Given that both the cold dust and the stars most efficient at heating dust are concentrated in the spiral arms, it is likely that those stars are the dominant heating source for the $160 \mu \mathrm{m}$ dust in the spiral arms.

\subsection{Infrared-Radio Correlation}

The far-IR dust emission and the radio continuum emission both trace active star formation in galaxies and are known to be tightly correlated on galactic scales (de Jong et al. 1985; Helou et al. 1985). In Figure 6, we show the far-IR (42$122 \mu \mathrm{m}$, FIR) to radio ratio map of M81 constructed using the $160 \mu \mathrm{m}$ resolution MIPS images and $20 \mathrm{~cm}$ continuum data $\left[q \equiv \log \left(\mathrm{FIR} / 3.75 \times 10^{-12} \mathrm{~Hz}\right)-\log \left(S_{1.49 \mathrm{GHz}}\right)\right]$. The MIPS bands were combined to create a TIR image, and the fractional 

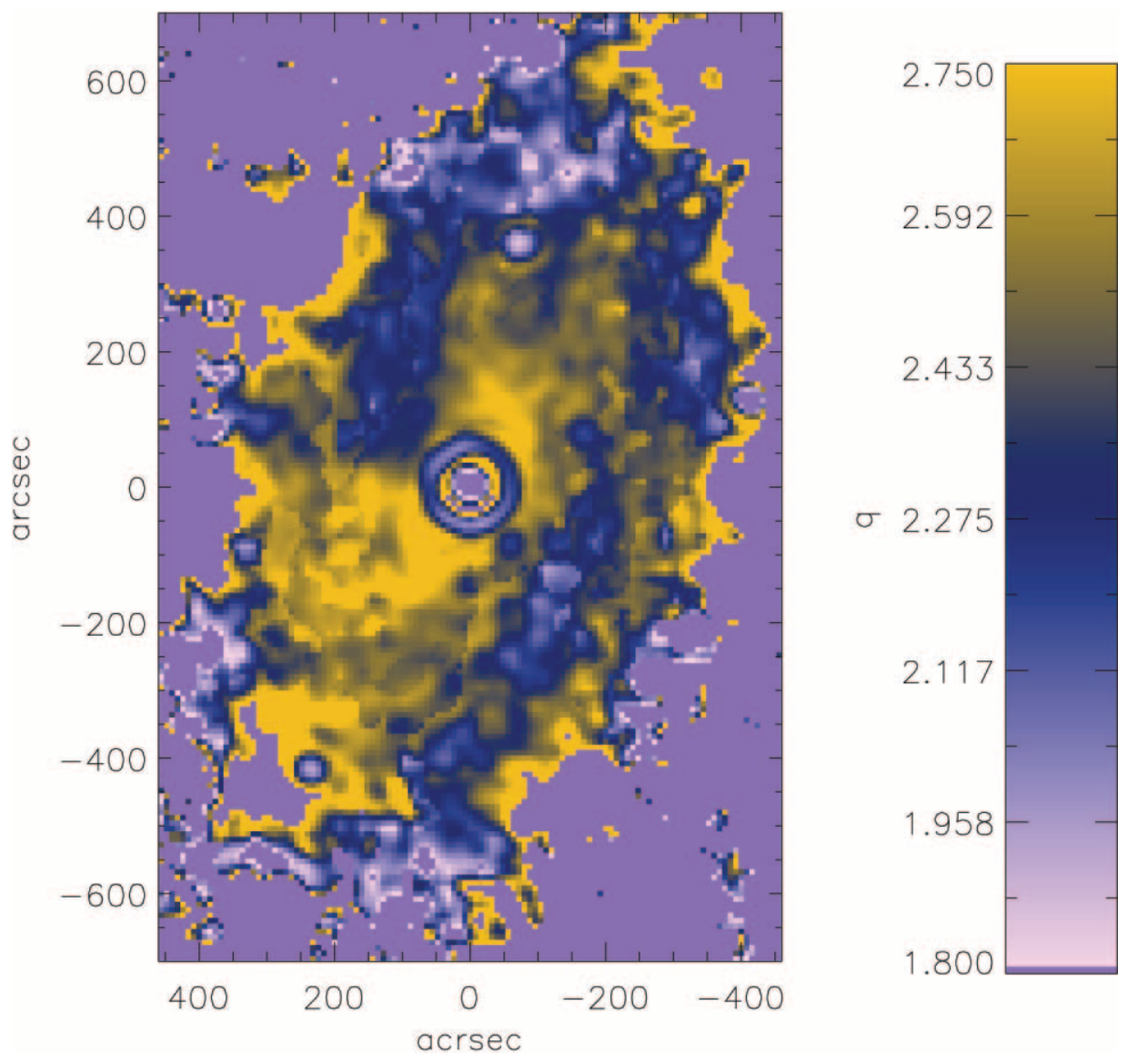

FIG. 6.-FIR to radio ratio $(q)$ map. This map probes the galaxy disk with a physical scale of $\sim 645$ pc. The field of view of the image is $15^{\prime} \times 23^{\prime}$.

FIR (Helou et al. 1985) component was estimated per pixel using the Dale \& Helou (2002) models. The value of $q$ ranges from $\sim 1.80$ for the AGN-dominated nuclear region to $\sim 2.87$ for the bright star-forming regions, with an intermediate value of $\sim 2.07$ for the interarm regions. A similar range in $q$ was seen for M33 by Hippelein et al. (2003), although they did not comment on any large-scale structure seen in $q$ variations as we see in M81. For comparison, the average $q$ value found for galaxies by Yun et al. (2001) is 2.34. The variation in $q$ is coherent with structures related to the M81 spiral arms, confirming that the radio-infrared correlation is more complex inside individual galaxies than between galaxies. This variation will be investigated in detail in future papers to explore the dynamics of cosmic-ray electron propagation and escape (Helou \& Bicay 1993).

\section{CONCLUSIONS}

We present MIPS images of M81 at 24, 70, and $160 \mu \mathrm{m}$ that reveal a bright nucleus and two well-resolved spiral arms studded with bright regions of star formation. These images show that M81 has a significant amount of cold dust associated with the spiral arms. From multiwavelength morphology comparisons, the dust heating is argued be dominated by recent star formation even at the longest MIPS wavelength. The resolved UV and $\mathrm{H} \alpha$ SFRs are always lower than the IR SFRs indicating significant dust attenuation, radiative transfer effects, and/or different stellar ages than assumed for the Kennicutt (1998) SFR calibrations. The character of the dust attenuation indicates that the dust geometry and/or grain properties are different for resolved regions in M81 than in starburst galaxies. The infrared-radio correlation was found to vary by a factor of $\sim 6$ in the M81 with coherent structures relating to the spiral arms. These results illustrates the need for additional theoretical and empirical work on how to accurately combine different SFR indicators, accounting for dust, age, and radiative transfer effects, to give an accurate view of the star formation in regions of galaxies.

The SINGS team is warmly thanked for allowing M81 to be part of the ERO program. We thank Rene Waterbos for providing us with the $\mathrm{H} \alpha$ and $R$-band images. The radio image used in this paper was taken with the VLA, which is operated by the National Radio Astronomy Observatory. This work is based on observations made with the Spitzer Space Telescope, which is operated by the Jet Propulsion Laboratory, California Institute of Technology, under NASA contract 1407. Support for this work was provided by NASA through contract 960785 issued by JPL/Caltech.
Appleton, P. N., Davies, R. D., \& Stephenson, R. J. 1981, MNRAS, 195, 327 Bell, E. F. 2003, ApJ, 586, 794

Bell, E. F., Gordon, K. D., Kennicutt, R. C., \& Zaritsky, D. 2002, ApJ, 565, 994

Bell, E. F., \& Kennicutt, R. C. 2001, ApJ, 548, 681

Boyce, P. J., et al. 2001, ApJ, 560, L127
Braun, M., Assendorp, R., Bontekoe, D. J. M., Kester, T. R., \& Richter, G. 1998, in The Magellanic Clouds and Other Dwarf Galaxies, ed. T. Richtler \& J. M. Braun (Aachen: Shaker), 121

Condon, J. J. 1992, ARA\&A, 30, 575

Dale, D. A., \& Helou, G. 2002, ApJ, 576, 159 
de Jong, T., Klein, U., Wielebinski, R., \& Wunderlich, E. 1985, A\&A, 147, L6

Devereux, N. A., Jacoby, G., \& Ciardullo, R. 1995, AJ, 110, 1115

Engelbracht, C. W., et al. 2004, ApJS, 154, 248

Fazio, G. H., et al. 2004, ApJS, 154, 10

Fioc, M., \& Rocca-Volmerange, B. 1997, A\&A, 326, 950

Freedman, W. L., et al. 2001, ApJ, 553, 47

Gordon, K. D., Clayton, G. C., Witt, A. N., \& Misselt, K. A. 2000, ApJ, 533, 236

Gordon, K. D., et al. 2004, PASP, submitted

Greenawalt, B., Walterbos, R. A. M., Thilker, D., \& Hoopes, C. G. 1998, ApJ, 506,135

Haarsma, D. B., Partridge, R. B., Windhorst, R. A., \& Richards, E. A. 2000, ApJ, 544, 641

Haas, M., Lemke, D., Stickel, M., Hippelein, H., Kunkel, M., Herbstmeier, U., \& Mattila, K. 1998, A\&A, 338, L33

Helou, G., \& Bicay, M. D. 1993, ApJ, 415, 93

Helou, G., Soifer, B. T., \& Rowan-Robinson, M. 1985, ApJ, 298, L7

Hill, J. K., et al. 1995, ApJ, 438, 181

Hippelein, H., Haas, M., Tuffs, R. J., Lemke, D., Stickel, M., Klaas, U., \& Völk, H. J. 2003, A\&A, 407, 137

Kaufman, M., Bash, F. N., Kennicutt, R. C., \& Hodge, P. W. 1987, ApJ, 319,61
Kennicutt, R. C. 1998, ARA\&A, 36, 189

Kennicutt, R. C., et al. 2003, PASP, 115, 928

Kewley, L. J., Geller, M. J., Jansen, R. A., \& Dopita, M. A. 2002, AJ, 124, 3135

Kong, X., Charlot, S., Brinchmann, J., \& Fall, S. M. 2004, MNRAS, 349, 769

Kong, X., et al. 2000, AJ, 119, 2745

Leitherer, C., et al. 1999, ApJS, 123, 3

Marcum, P. M., et al. 2001, ApJS, 132, 129

Meurer, G. R., Heckman, T. M., \& Calzetti, D. 1999, ApJ, 521, 64

Pierini, D., Popescu, C. C., Tuffs, R. J., \& Völk, H. J. 2003, A\&A, 409, 907

Rosa-González, D., Terlevich, E., \& Terlevich, R. 2002, MNRAS, 332, 283

Rieke, G. H., et al. 2004, ApJS, 154, 25

Schlegel, D. J., Finkbeiner, D. P., \& Davis, M. 1998, ApJ, 500, 525

Stecher, T. P., et al. 1997, PASP, 109, 584

Swartz, D. A., Ghosh, K. K., McCollough, M. L., Pannuti, T. G., Tennant, A. F., \& Wu, K. 2003, ApJS, 144, 213

Werner, M. W., et al. 2004, ApJS, 154, 1

Wilke, K., Stickel, M., Haas, M., Herbstmeier, U., Klaas, U., \& Lemke, D. 2003, A\&A, 401, 873

Willner, S., et al. 2004, ApJS, 154, 222

Witt, A. N., \& Gordon, K. D. 2000, ApJ, 528, 799

Yun, M. S., Ho, P. T. P., \& Lo, K. Y. 1994, Nature, 372, 530

Yun, M. S., Reddy, N. A., \& Condon, J. J. 2001, ApJ, 554, 803 


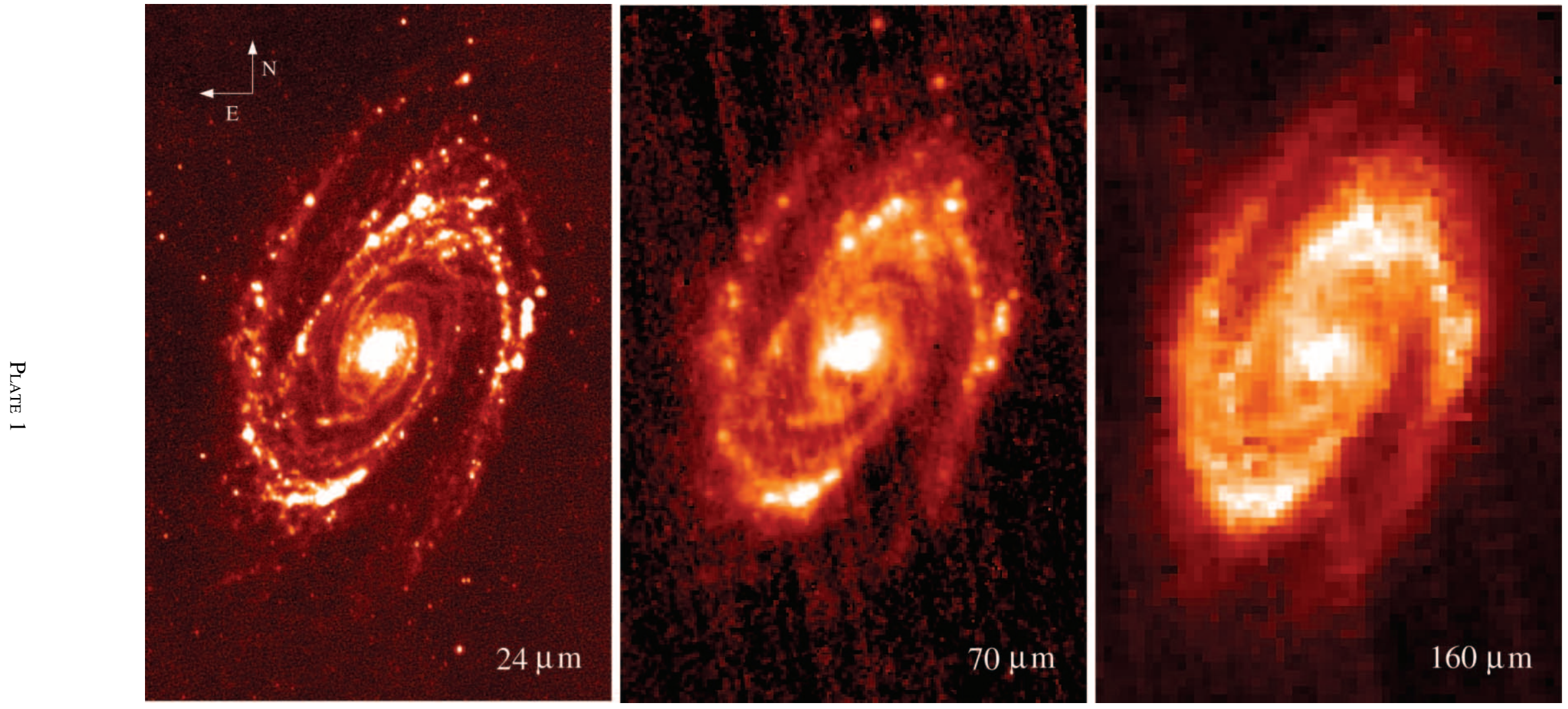

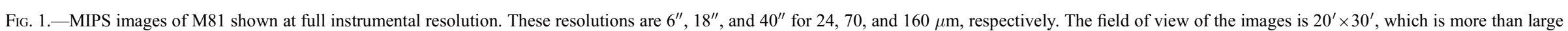
enough to cover M81. These mosaics have a total exposure time of approximately 80,40 , and $8 \mathrm{~s}$ per point at 24,70 , and $160 \mu \mathrm{m}$, respectively. 


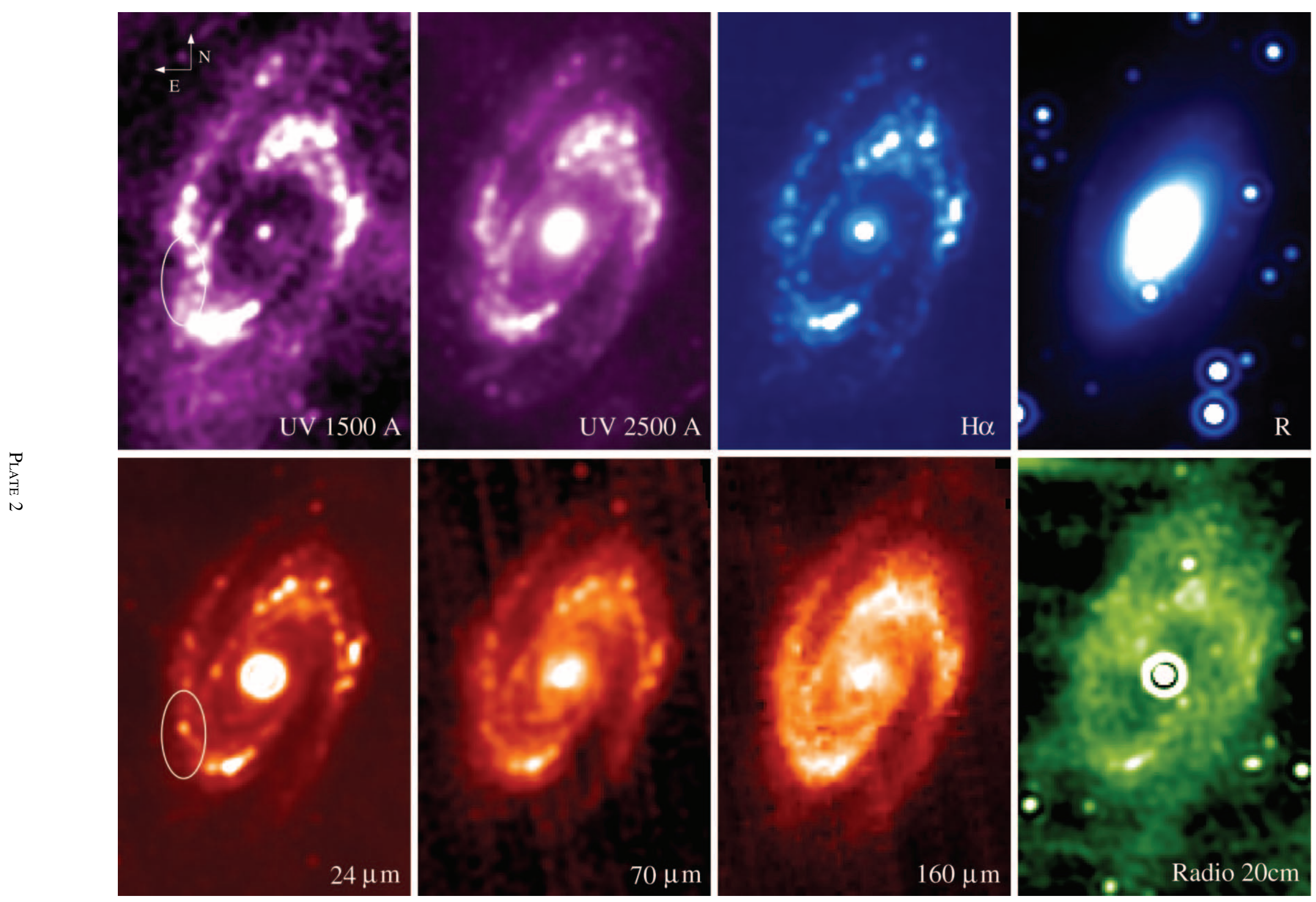

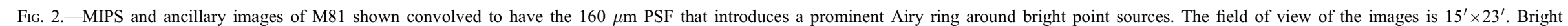
foreground stars were masked in the UV and $\mathrm{H} \alpha$ images prior to convolution. The ellipse denotes the region discussed in $\S 3.1$. 